\title{
H.E.S.S. precision measurements of the SNR RX J1713.7-3946
}

\section{Eger, P.*}

Max-Planck-Institut für Kernphysik, Heidelberg

E-mail: peter.eger@mpi-hd.mpg.de

Parsons, R.D.

Max-Planck-Institut für Kernphysik, Heidelberg

E-mail: daniel.parsons@mpi-hd.mpg.de

Berge, D.

GRAPPA; Univ. of Amsterdam

E-mail: D.Berge@uva.nl

Zabalza, V.

Univ. of Leicester

E-mail: victor.zabalza@leicester.ac.uk

\section{Aharonian, $\mathbf{F}$.}

Max-Planck-Institut für Kernphysik, Heidelberg; Dublin Institute for Advanced Studies E-mail: aharon@mpi-hd.mpg.de

\section{Funk, S.}

Univ. Erlangen-Nürnberg, ECAP

E-mail: s.funkefau.de

\section{Uchiyama, Y.}

Rikkyo Univ.

E-mail: y.uchiyama@rikkyo.ac.jp

\section{Bryan, $\mathbf{M}$.}

GRAPPA, Univ. of Amsterdam

E-mail: M.P.Bryan@uva.nl

on behalf of the H.E.S.S. Collaboration 
The shell-type supernova remnant RXJ1713.7-3946 is one of the brightest TeV gamma-ray sources in the Galaxy detected by the High Energy Stereoscopic System (H.E.S.S.). Despite extensive multi-wavelength coverage in gamma-rays, X-rays and lower energy regimes, the nature of the underlying gamma-ray radiation mechanisms is still under debate. Here we present new precision measurements obtained with the H.E.S.S. array in its 4-telescope configuration, based on 150 hours of observations and the usage of more sensitive analysis techniques. The new results feature an improvement in the exposure by factors of 2 (sky images) to 4 (spectra) over previous measurements, allowing for spectral and morphological studies at unprecedented precision, and yielding the most detailed $\mathrm{TeV}$ gamma-ray analysis of any extended object in the sky. The angular resolution, better than $0.05 \mathrm{deg}$, enables for the first time a detailed investigation of morphological differences between $\mathrm{TeV}$ gamma rays and $\mathrm{X}$-rays.

The 34th International Cosmic Ray Conference, 30 July- 6 August, 2015

The Hague, The Netherlands

${ }^{*}$ Speaker. 
Further details will be available in an upcoming publication by the H.E.S.S. Collaboration. 